\title{
Characterization of Elastic Strain Field and Geometrically Necessary Dislocation Distribution in Stress Corrosion Cracking of 316 Stainless Steels by Transmission Kikuchi Diffraction
}

\author{
Arantxa Vilalta-Clemente ${ }^{1}$, Martina Meisnar ${ }^{1}$, Sergio Lozano-Perez ${ }^{1}$, and Angus J. Wilkinson ${ }^{1}$. \\ 1. Department of Materials, University of Oxford, Parks Road, Oxford OX1 3PH, United Kingdom.
}

Stainless steel alloys such as SUS 316 are widely used for the application in nuclear power plants because of their excellent performance in high-temperature and corrosive environments. In this work, a stress corrosion crack from a sample tested under simulated primary water from a pressurized water reactor has been characterized.

Transmission electron backscattered diffraction (t-EBSD) or transmission Kikuchi diffraction (TKD) in the scanning electron microscope (SEM) is emerging as a very promising approach to characterize materials at the nano-scale. TKD involves the analysis of electron transparent samples, similar to those prepared for transmission electron microscopy (TEM). Due to smaller interaction volume of the incident beam with the specimen, the spatial resolution provided by TKD can be below $10 \mathrm{~nm}$, which is a significant improvement over conventional EBSD [1-3].

The TKD geometry is derived from the basic EBSD setup with the main difference being the specimen position and its orientation with respect to the SEM column. The thin foil sample is kept almost horizontal $\left(\sim 10^{\circ}\right.$ tilt $)$ with respect to the incident electron beam, as opposed to the $70^{\circ}$ tilt required for conventional EBSD. A Zeiss Merlin Field-Emission SEM equipped with an EBSD Bruker e-Flash HR detector with Forescatter Diodes (FSDs) below the phosphor screen was used for the data acquisition. The ability to alter the camera tilt provides additional flexibility in setting up the geometry. In this work, we have analyzed TKD patterns using the cross-correlation algorithms presented by Wilkinson et al [4] initially for high angular resolution analysis of EBSD patterns. As in EBSD, changes in elastic strain and lattice rotations cause small shifts in the positions of zones axes and other features in the TKD patterns. Cross-correlation is used to measure the variations between each test pattern and a reference pattern in at least 35 sub-regions distributed across the pattern. The dispersion of shifts across the pattern is used to determine the change in lattice strain and rotation relative to the reference point.

Figure1a was obtained using colour coding of the signals from the FSDs and provides information on the orientation contrast within the stainless steel sample, and reveals the location of the crack, the misorientation of the grains on either sides of the crack, the existence of twinning deformation and slip. TKD maps were obtained using $11 \mathrm{~nm}$ step size, with the detector binned to record patterns at 160 by 120 pixels. We have used the cross correlation method to study the deformation around the crack tip.

Example maps for a selected strain component ( $\varepsilon_{12}$ shear strain) and two rotation components $\left(\omega_{12}\right.$ about the sample normal, and $\omega_{13}$ about the vertical axis) are shown in Figure 2. The in-plane shear strain $\varepsilon_{12}$ distribution shows a maximum in the top grain, at the intersection of the grain boundary with the deformation bands. The $\omega_{12}$ lattice rotations are shown in Figure $2 b$, where larger values are observed in the region below the crack in the bottom grain and between the deformation bands in the top grain.

We have used the resulting rotation tensor and the Nye geometrically necessary dislocations (GNDs) to 
assess the dislocation content in the 316 Stainless Steel sample. Accumulation of GNDs on the deformation bands, twining and slip bands as well near the open crack between the two grains and the grain boundary itself is observed (Figure 1b). The deformation history of this type of samples indicates that the band intersecting the grain boundary is deformed bands with high dislocation density.

Furthermore, TKD has been used to measure the grain boundary orientation and establish a gauge for a quantifying plastic deformation at the crack tip and other regions in the surrounding matrix [5].

\section{References:}

[1] R R Keller and R H Geiss, J. Microsc 245 (2012), p. 245.

[2] P W Trimby, Ultramicroscopy 120 (2012), p. 16.

[3] P W Trimby et al, Acta Materialia 62 (2014), p. 69.

[4] A J Wilkinson et al, Ultramicroscopy 106 (2006), p. 307.

[5] M M et al, Journal of Nuclear Materials (submitted).

[6] The authors acknowledge funding from EPSRC grant No: EP/J015792/1 \& EP/J016098/1 and Areva (UK). Samples were provided by INSS (Japan).
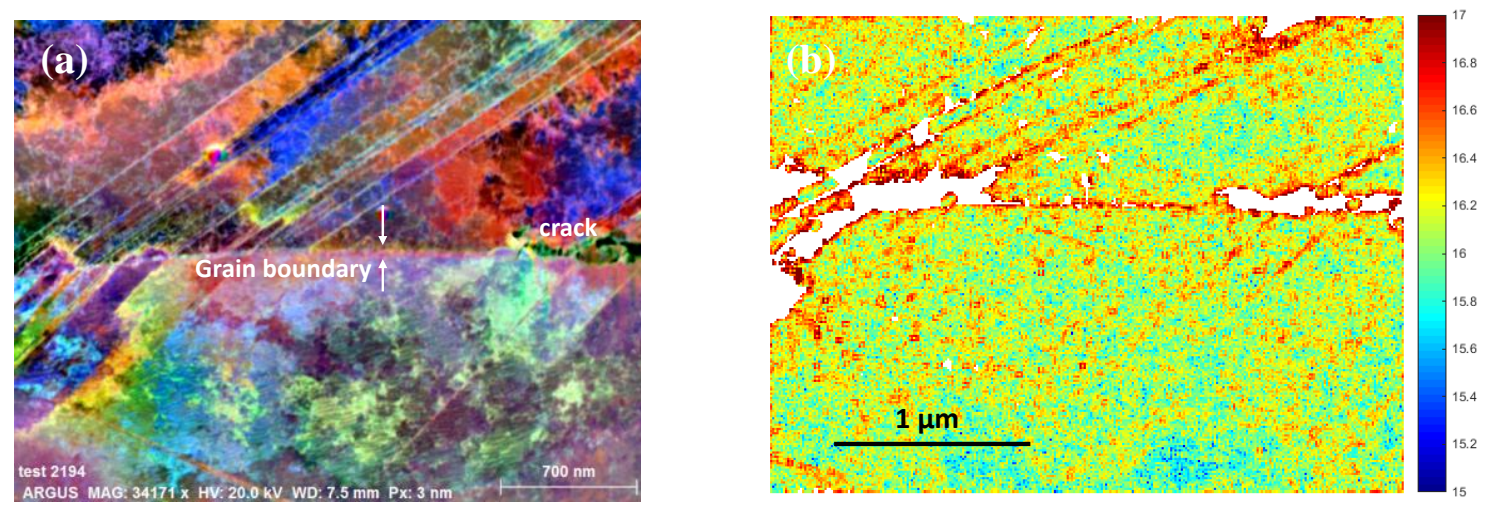

Figure 1. (a) FSE image showing the crack and the grain boundary (b) GND density map generated using $11 \mathrm{~nm}$ step size and the colour scale is dislocations per $\mathrm{m}^{2}$ on a $\log _{10}$ scale.
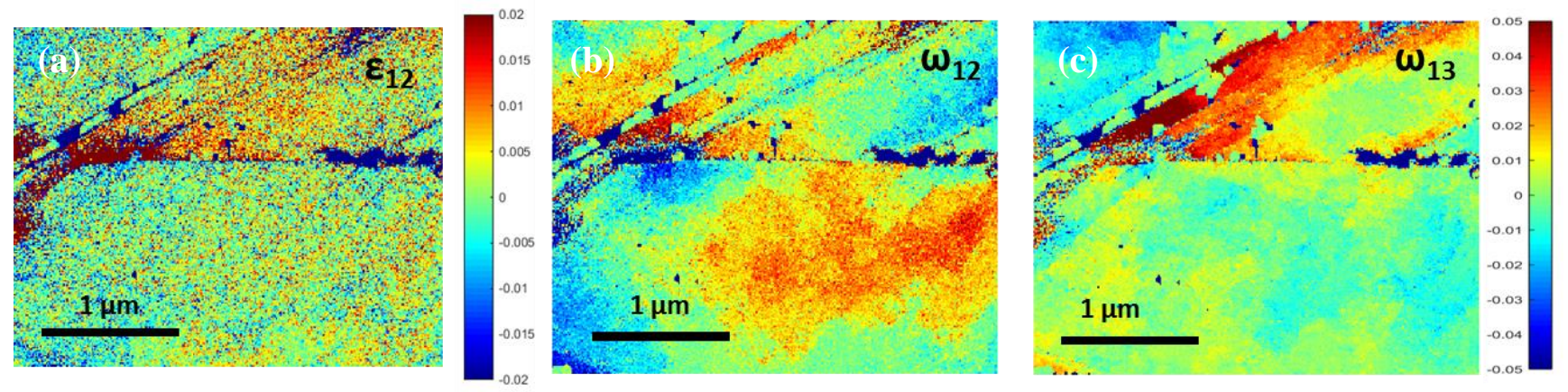

Figure 2. Example strain and rotations. 